\title{
Etude de l'endoparasitisme expérimental de Trichomonas vaginalis chez la Souris
}

III. Essais d'installation d'une souche réfractaire

de Tricbomonas vaginalis. Interprétation des résultats.

par J.-G. GOBERT, P. GEORGES et J. SAVEL

[Laboratoire de Parasitologie, Faculté de Pharmacie de Paris (Professeur Cavier), 4, avenue de l'Observatoire, F 75 - Paris-6 $\left.6^{\circ}\right]$

\section{Résumé}

Dans ce travail sont rapportés les essais d'installation, chez la Souris, d'une souche de Trichomonas vaginalis que le vieillissement, au cours d'une culture prolongée in vitro, avait rendu réfractaire à toute implantation chez l'animal.

Seule, l'addition au milieu de culture, dans lequel cette souche est entretenue, d'un homogénéisat de flagellés de même espèce, de souche différente et beaucoup plus jeunes, est capable de provoquer une exaltation provisoire du caractère agressif qui se traduit par la possibilité d'implantation chez l'animal avec développement fréquent «d'abcès à formes latentes ».

De plus, il a été prouvé que le séjour chez l'animal n'est pas une condition suffisante pour obtenir l'acquisition durable du caractère virulent, comme le prouvent les résultats de réinoculations pratiquées à partir des abcès contenant des flagellés mobiles ou des formes latentes.

\section{Summary}

The authors report the results of mice infestations trials with an old strain of Trichomonas vaginalis, which a prolonged culture rendered unable to any implantation in animals.

The addition to the culture in which the strain has been maintained of an homogeneisat of the same species of Flagellates, taken from different and much younger strains, may restore a temporary exaltation of the virulence; it revealed the capacity of the culture of infesting animals with frequent development of «latent forms of abcess ». 
Moreover, it has been prouved by reinoculations made with samples taken in abcess conaaining living Flagellates or latent forms, that the implantation in animals of these Flagellates is not a sufficient condition to restore durably the character of virulence.

\section{Introduction.}

Il nous a été possible, dans un travail antérieur (2), de vérifier, à l'aide de différentes souches de Trichomonas vaginalis, l'exactitude d'une constatation expérimentale signalée par Inoki (4), selon laquelle l'injection intraveineuse d'hématies de Poulet à des souris permet d'implanter, de manière durable, Trichomonas vaginalis ultérieurement injecté, par voie intrapéritonéale, à ces rongeurs. Les flagellés sont retrouvés, le plus souvent, au niveau du foie et du pancréas et se montrent, après réinjection à des souris neuves, directement infectieux et capables de déterminer des lésions viscérales qui ont fait l'objet d'une récente publication (3).

Cependant, nous avons pu observer que l'une de nos souches, répertoriée S. 8 , entretenue depuis onze ans, au laboratoire, en culture sur milieu de Magara (5), se montrait absolument réfractaire à une quelconque implantation chez l'animal, malgré le traitement préalable des souris par des hématies de Poulet. Or, en 1961, la même souche avait donné, à l'un d'entre nous (1), des résultats conformes à ceux d'Inoki.

Nous rapportons ici les tentatives qui ont eu pour but de conférer ou de rendre à la souche S. 8 la possibilité de s'implanter chez la Souris et d'y développer un pouvoir infectieux.

\section{Principes.}

Nous avons déjà observé et décrit (2) que l'endoparasitisme de Trichomonas vaginalis chez la Souris se manifeste d'abord au niveau du pancréas et au niveau du foie. Ce tropisme pancréatique et hépatique nous permettait d'envisager la possibilité de créer un pouvoir virulent chez les flagellés en les cultivant, in vitro, en présence d'un broyat de pancréas ou de foie de Souris. Certaines souches, S. 12, S. 13, S. 14, traitées dans ces conditions, nous avaient d'ailleurs valu des résultats encourageants (6).

D'autre part, nous avions prouvé la possibilité de transmission, de génération à génération, du caractère virulent acquis par certaines souches (2). Nous avons alors cherché à savoir si la culture de la souche S. 8 en présence d'un homogénéisat de flagellés appartenant à la souche S. 12, naturelle ou virulente, pouvait faire réapparaître, chez la souche S. 8, un ou plusieurs caractères qu'elle avait apparemment perdus au cours de sa longue conservation en culture.

Nous avons également pensé à combiner les deux traitements prédécents en cultivant la souche S. 8 en présence à la fois d'un broyat pancréatique ou hépatique et d'homogénéisat de la souche S. 12.

Toutes les expériences fondées sur ces trois principes ont été menées de front. 
Enfin, nous avons étudié le comportement de la souche S. 8, traitée selon les modalités précédentes et ensuite inoculée à des souris préalablement préparées, soit par des hématies d'embryon de poulet, soit par l'azathioprine.

Il est important de signaler, dès maintenant, que cette dernière partie de notre travail est intervenue plus tardivement, au vu de l'évolution des résultats observés chez les animaux non préparés.

\section{Méthodes expérimentales :}

\section{A. Traitement de la souche S. 8 :}

\section{Par addition de broyat, pancréatique ou hépatique:}

Nous réalisons, à 48 heures d'intervalle, trois repiquages successifs des flagellés de la souche S. 8 sur du milieu de Magara. Au moment de chaque repiquage, nous ajoutons $0,5 \mathrm{ml}$ d'une suspension obtenue par trituration, au mortier, de pancréas ou de foie de Souris, fraîchement prélevé, à raison de $1 \mathrm{~g}$ de tissu frais pour $3 \mathrm{ml}$ de soluté isotonique ; ces opérations sont menées aseptiquement.

Ultérieurement, les repiquages sont régulièrement poursuivis dans du milieu de Magara auquel aucun broyat tissulaire n'est alors ajouté.

Les souches ainsi traitées sont désignées, dans la suite de notre texte, sous les appellations $S .8+$ foie ou $S .8+$ pancréas.

Les animaux à infester, groupés le plus souvent par lots de dix individus, reçoivent :

- soit un inoculum provenant de la troisième culture menée en présence de broyat tissulaire ;

- soit un inoculum provenant des cultures ultérieures menées sans addition de broyat. Dans ce cas, une série d'animaux est inoculée chaque semaine.

\section{Par addition d'un homogénéisat de Trichomonas vaginalis de souche différente:}

On réalise, à 48 heures d'intervalle, trois repiquages successifs des flagellés de la souche S. 8 sur du milieu de Magara. Au moment de chaque repiquage, nous ajoutons $1 \mathrm{ml}$ de suspension de matériel cellulaire provenant du broyage mécanique, à l'aide de billes de verre ${ }^{*}$, d'une suspension de $150 \times 10^{6}$ flagellés par ml. L'efficacité de l'opération est constatée par plusieurs cultures de la suspension obtenue, maintenue à $37^{\circ} \mathrm{C}$ pendant quinze jours. Aucun développement n'a été observé dans ces tubes de contrôle.

Ultérieurement, les repiquages sont régulièrement poursuivis dans du milieu de Magara auquel aucun homogénéisat n'est alors ajouté.

Les souches ainsi traitées sont désignées, dans la suite de notre texte, sous les appellations $S .8+S .12$ naturel ou $S .8+S .12$ virulent.

- Appareillage Vibrogène (Buhl) avec des billes de calibre 31/9; temps: 7 minutes. 
Les animaux à infester, groupés le plus souvent par lots de dix individus, reçoivent :

— soit un inoculum provenant de la troisième culture menée en présence d'homogénéisat ;

- soit un inoculum provenant des cultures ultérieures menées sans addition d'homogénéisat. Dans ce cas, une série d'animaux est inoculée chaque semaine.

3. Par addition d'homogénéisat de Trichomonas vaginalis, souche S. 12 naturel et de broyat pancréatique ou hépatique:

Dans un premier temps, la souche S. 8 subit d'abord trois repiquages successifs en présence d'homogénéisat de la souche S. 12, puis, dans un second temps, trois repiquages successifs en présence de broyat d'origine pancréatique ou hépatique.

Ultérieurement, les repiquages sont régulièrement poursuivis dans le milieu de Magara où n'est plus ajouté ni broyat tissulaire, ni homogénéisat de protozoaires.

Les souches ainsi traitées sont désignées, dans la suite de notre texte $S .8+$ S. 12 + foie ou $S .8+$ S. $12+$ pancréas.

Les animaux à infester, groupés le plus souvent par lot de dix individus, reçoivent un inoculum provenant des cultures terminales menées sans addition d'aucune sorte. Une série d'animaux est inoculée chaque semaine.

Dans tous les cas (1. 2. et 3), 250.000 flagellés ont été injectés à chaque animal.

B. Traitement des souris.

Nous utilisons des souris femelles Swiss Webster C FI, de race pure et exemptes de contamination bactérienne. Elles sont âgées de six semaines au moment de l'injection des flagellés et pèsent alors environ $20 \mathrm{~g}$.

Les traitements préparants, lorsqu'ils ont été réalisés, font appel, soit à l'injection intra-veineuse de $1,5 \times 10^{6}$ hématies d'embryon de Poulet, soit à l'ingestion d'azathioprine à raison de $3 \mathrm{mg}$ par $\mathrm{kg}$ et par jour, pendant trois jours. Pour certains lots, ces deux modes de préparation ont été jumelés.

L'injection de Trichomonas vaginalis, par voie intra-péritonéale, intervient entre 48 et 72 heures après la fin du traitement préparant.

Dans tous les cas, les animaux sont sacrifiés au bout de trois semaines, délai maximum d'apparition de lésions graves chez les animaux dont l'infestation a été réussie.

\section{IV. - Résultats :}

Les abcès qui peuvent être observés chez les animaux infestés sont, dans la plupart des cas, localisés au niveau du foie ou du pancréas. Toujours de petite taille, ils sont tous examinés au microscope, après écrasement entre lame et lamelle. Cet examen permet de distinguer deux catégories d'abcès: la première groupe ceux où des flagellés vivants sont observables (abcès à formes mobiles), la seconde, ceux dont l'examen microscopique ne permet pas de retrouver de formes mobiles. 
Par ailleurs, tous les abcès de l'une ou de l'autre catégorie sont mis en culture sur milieu de Magara: cela permet, en ce qui concerne la seconde catégorie, de constater, soit l'échec de la culture, soit son développement: ce sont ces derniers abcès, fournissant une culture ultérieure positive que nous qualifions d'abcès à formes latentes, sans préciser pour l'instant la forme, différente du trophozoïte, que revêt le flagellé au sein de ces abcès.

Les résultats observés sont consignés dans les tableaux 1, 2, 3, 4 .

Enfin, les flagellés provenant des cultures d'abcès à formes mobiles, de loin les moins nombreux, ou d'abcès à formes latentes, ont été systématiquement injectés, par voie intra-péritonéale, à raison de 250.000 flagellés par animal, à des souris neuves n'ayant subi aucune préparation (Tableau 5).

Ces tableaux font apparaître le nombre total d'abcès et, parmi ceux-ci, le nombre d'abcès à formes à latentes.

\section{Tableau 1}

\begin{tabular}{|c|c|c|c|c|}
\hline SOUCHE & $\begin{array}{l}\text { Nombre DE } \\
\text { SOURIS }\end{array}$ & $\begin{array}{l}\text { NoMBRE DE } \\
\text { FLAGELLÉS } \\
\text { INJECTÉS }\end{array}$ & $\begin{array}{l}\text { NOMBRE TOTAL } \\
\text { D'ANIMAUX POR- } \\
\text { TEURS D'ABCÈS }\end{array}$ & $\begin{array}{c}\text { NOMBRE D'ANI- } \\
\text { MAUX PORTEURS } \\
\text { D'ABCĖS } \\
\text { A FORMES } \\
\text { LATENTES }\end{array}$ \\
\hline $\begin{array}{l}\text { S. } 8 \\
\text { (Souris non prépa- } \\
\text { rées). }\end{array}$ & $\begin{array}{r}5 \\
10 \\
20\end{array}$ & $\begin{array}{c}3,2 \text { millions } \\
125.000 \\
250.000\end{array}$ & $\begin{array}{l}1 \\
0 \\
0\end{array}$ & $\begin{array}{l}1 \\
0 \\
0\end{array}$ \\
\hline $\begin{array}{l}\text { S. } 8 \\
\text { (Souris préparées se- } \\
\text { lon Inoki) }\end{array}$ & $\begin{array}{r}5 \\
15 \\
20\end{array}$ & $\begin{array}{c}3,5 \text { millions } \\
500.000 \\
300.000\end{array}$ & $\begin{array}{l}0 \\
0 \\
0\end{array}$ & $\begin{array}{l}0 \\
0 \\
0\end{array}$ \\
\hline
\end{tabular}

Tableau 2

\begin{tabular}{|c|c|c|c|c|}
\hline Souche & $\begin{array}{l}\text { NombRE DE } \\
\text { SOURIS }\end{array}$ & $\begin{array}{l}\text { NOMBRE DE } \\
\text { FLAGELLÉS } \\
\text { INJECTÉS }\end{array}$ & $\begin{array}{l}\text { NOMBRE } \\
\text { D'ABCÈS }\end{array}$ & $\begin{array}{c}\text { NOMBRE D'ABCÈS } \\
\text { A FORMES } \\
\text { LATENTES }\end{array}$ \\
\hline S. $8+$ foie & 30 & 250.000 & 1 & 0 \\
\hline S. $8+$ pancréas & 29 & 250.000 & 1 & 0 \\
\hline
\end{tabular}

\section{Interprétation mathématique et discussion.}

Un certain nombre d'animaux avaient été inoculés avec la souche S. 8 naturelle ou les souches S. 8 traitées depuis plus de quatre semaines. Dans ces conditions, il 
nous est rapidement apparu que, passé ce délai, les flagellés traités avaient perdu toute virulence. Aussi n'avons-nous pas reporté dans les tableaux 1,2 et 3 , les résultats concernant les animaux injectés au cours de cette période et qui se sont tous révélés négatifs.

Tableau 3

\begin{tabular}{|c|c|c|c|c|}
\hline SOUCHE & $\begin{array}{l}\text { NoMBRE DE } \\
\text { SOURIS }\end{array}$ & $\begin{array}{l}\text { NOMBRE DE } \\
\text { FLAGELLÉS } \\
\text { INJECTÉS }\end{array}$ & $\begin{array}{l}\text { NOMBRE } \\
\text { D'ABCÈS }\end{array}$ & $\begin{array}{c}\text { NOMBRE D'ABCÈS } \\
\text { A FORMES } \\
\text { IATENTES }\end{array}$ \\
\hline S. $8+$ S. 12 nat. & 45 & 250.000 & 7 & 4 \\
\hline $\begin{array}{l}\text { S. } 8+\text { S. } 12 \text { nat. } \\
+ \text { foie }\end{array}$ & 45 & 250.000 & 4 & 3 \\
\hline $\begin{array}{l}\text { S. } 8+\text { S. } 12 \text { nat. } \\
+ \text { pancréas }\end{array}$ & 45 & 250.000 & 3 & 2 \\
\hline S. $\underset{\text { lent }}{8}+$ S. 12 viru- & 73 & 250.000 & 18 & 6 \\
\hline
\end{tabular}

Tableau 4

\begin{tabular}{|c|c|c|c|c|}
\hline SOUCHE & $\begin{array}{l}\text { NombRE DE } \\
\text { SOURIS }\end{array}$ & $\begin{array}{l}\text { NOMBRE DE } \\
\text { FLAGELLÉS } \\
\text { INJECTÉS }\end{array}$ & $\begin{array}{l}\text { NoMBRE } \\
\text { D'ABCÈS }\end{array}$ & $\begin{array}{c}\text { NOMBRE D'ABCÈS } \\
\text { A FORMES } \\
\text { LATENTES }\end{array}$ \\
\hline $\begin{array}{l}\text { S. } 8+\text { S. } 12 \text { souris } \\
\text { préparées }\end{array}$ & 117 & 250.000 & 32 & 4 \\
\hline
\end{tabular}

Tableau 5

\begin{tabular}{|c|c|c|c|c|}
\hline SOUCHE & $\begin{array}{l}\text { NombRE DE } \\
\text { SOURIS }\end{array}$ & $\begin{array}{l}\text { NOMBRE DE } \\
\text { FLAGELLÉS } \\
\text { INJECTÉS }\end{array}$ & $\begin{array}{l}\text { NOMBRE } \\
\text { D'ABCÈS }\end{array}$ & $\begin{array}{c}\text { NOMBRE D'ABCÈs } \\
\text { A FORMES } \\
\text { LATENTES }\end{array}$ \\
\hline $\begin{array}{l}\text { S. } 8 \text { provenant d'ab- } \\
\text { cès à formes la- } \\
\text { tentes }\end{array}$ & 107 & 250.000 & 10 & 1 \\
\hline
\end{tabular}

Les résultats ont pu être regroupés en raison de l'homogénéité des constatations expérimentales effectuées au niveau de chaque lot d'animaux.

Ainsi, les tableaux 1 et 2 montrent que, au cours des 14 premières séries d'essais portant sur 134 animaux, il n'a été observé qu'un seul abcès à formes latentes. 
En revanche, dès que l'on procède à l'injection de flagellés S. 8 cultivés en présence d'un homogénéisat de Trichomonas, les tableaux 3 et 4 font apparaître systématiquement, pour les 21 premières séries d'essais, portant sur 208 animaux, un nombre d'abcès bien plus élevé.

Enfin, pour le tableau 5, le nombre total d'abcès obtenus dans deux séries expérimentales (107 animaux) mérite attention.

Des comparaisons ont été ainsi effectuées entre les différents lots d'animaux présentés dans le tableau récapitulatif 6 .

Tableau 6

\begin{tabular}{|c|c|c|c|c|}
\hline SOUCHE & $\begin{array}{l}\text { NoMBRE DE } \\
\text { SOURIS }\end{array}$ & $\begin{array}{l}\text { NOMBRE DE } \\
\text { FLAGELLÉS } \\
\text { INJECTÉS }\end{array}$ & $\begin{array}{l}\text { NOMBRE TOTAL } \\
\text { D'ANIMAUX POR- } \\
\text { TEURS D'ABCÈS }\end{array}$ & $\begin{array}{l}\text { NOMBRE D'ANI- } \\
\text { MAUX PORTEURS } \\
\text { D'ABCÈS A FOR- } \\
\text { MES LATENTES }\end{array}$ \\
\hline $\begin{array}{l}\text { S. } 8 \text { seul } \\
\text { S. } 8+\text { foie ou S. } 8 \\
+ \text { pancréas }\end{array}$ & 134 & 250.000 & 3 & 1 \\
\hline $\begin{array}{l}\text { S. } 8+\mathrm{S} .12 \text { (souris } \\
\text { non préparées) }\end{array}$ & 208 & 250.000 & 30 & 15 \\
\hline $\begin{array}{l}\text { S. } 8+\mathrm{S} .12 \text { (souris } \\
\text { préparées) }\end{array}$ & 117 & 250.000 & 32 & 4 \\
\hline $\begin{array}{l}\text { S. } 8 \text { provenant d'ab- } \\
\text { cès à formes la- } \\
\text { tentes }\end{array}$ & 107 & 250.000 & 10 & 1 \\
\hline
\end{tabular}

Le calcul du $\chi^{2}$, en permettant de comparer deux à deux les différents lots expérimentaux inoculés au cours des 4 premières semaines d'expérimentation doit permettre d'apprécier la signification des résultats.

\section{A. SOURIS NON PRÉPARÉES.}

1. Comparaison du nombre total d'abcès obtenus, d'une part avec la souche S. 8 non traitée ou traitée par un broyat de tissu hépatique ou pancréatique, d'autre part avec la souche S. 8 traitée préalablement par un homogénéisat de flagellés, S. 12 seul ou associé à un broyat tissulaire (Tableau 7).

Le $\chi^{2}$ est voisin de 14 . Le problème étudié est à 1 degré de liberté; les tables donnent 3,84 avec un seuil de confiance de 0,95 et 6,64 avec un seuil de confiance de 0,99 . La différence est donc significative et la culture de flagellés S. 8 en présence d'un homogénéisat de la souche S. 12 semble conférer à cette souche S. 8 une virulence incontestable. 


\section{Tableau 7}

\begin{tabular}{|c|c|c|c|c|c|c|}
\hline SOUCHE & \multicolumn{2}{|c|}{$\begin{array}{c}\text { RÉSULTATS } \\
\text { EXPÉRIMENTAUX }\end{array}$} & $\begin{array}{l}\text { VALEURS } \\
\text { THÉORIQUES }\end{array}$ & ECARTS & $\begin{array}{l}\text { CARRÉ DES } \\
\text { ÉCARTS }\end{array}$ & $\begin{array}{l}\text { CARRE des / VALEUR } \\
\text { ECARTS }\end{array}$ \\
\hline $\begin{array}{l}\text { S. } 8 \text { seul et S. } 8 \\
+ \text { foie ou } \\
\text { pancréas }\end{array}$ & $\begin{array}{l}\text { Echecs } \\
\text { Abcès }\end{array}$ & $\begin{array}{r}131 \\
3\end{array}$ & $\begin{array}{r}121,14 \\
12,86\end{array}$ & $\begin{array}{l}9,86 \\
9,86\end{array}$ & $\begin{array}{l}97,22 \\
97,22\end{array}$ & $\begin{array}{l}0,80 \\
7,60\end{array}$ \\
\hline \multirow[t]{2}{*}{ S. $8+$ S. 12} & $\begin{array}{l}\text { Echecs } \\
\text { Abcès }\end{array}$ & $\begin{array}{r}178 \\
30\end{array}$ & $\begin{array}{r}188,03 \\
19,97\end{array}$ & $\begin{array}{l}10,03 \\
10,03\end{array}$ & $\begin{array}{l}100,6 \\
100,6\end{array}$ & $\begin{array}{l}0,54 \\
5,04\end{array}$ \\
\hline & $\begin{array}{l}\text { Total } \\
\text { Souris } \\
\text { Total } \\
\text { Abcès }\end{array}$ & $\begin{array}{r}342 \\
33\end{array}$ & & & & $\varkappa^{2}=13,98$ \\
\hline
\end{tabular}

2. Comparaison du nombre total d'abcès à formes latentes obtenus dans les deux mêmes lots que ci-dessus.

\section{Tableau 8}

\begin{tabular}{|c|c|c|c|c|c|}
\hline SOUCHE & $\begin{array}{l}\text { RÉSULTATS EX- } \\
\text { PÉRIMENTAUX }\end{array}$ & $\begin{array}{l}\text { VALEUR } \\
\text { THÉORIQUE }\end{array}$ & ECARTS & $\begin{array}{c}\text { CARRÉ DES } \\
\text { ÉCARTS }\end{array}$ & $\begin{array}{l}\text { CAKRE des / VALEUR } \\
\text { ECARTS } / \text { THEORIQUE }\end{array}$ \\
\hline $\begin{array}{l}\text { S. } 8 \text { seul et S. } 8 \\
+ \text { foie ou pan- } \\
\text { créas }\end{array}$ & $\begin{array}{l}\text { Echecs } \\
\text { Formes } \\
\text { latentes }\end{array}$ & $\begin{array}{r}127,3 \\
6,3\end{array}$ & $\begin{array}{l}5,7 \\
5,3\end{array}$ & $\begin{array}{l}32,5 \\
28,1\end{array}$ & $\begin{array}{l}0,22 \\
4,46\end{array}$ \\
\hline \multirow[t]{2}{*}{$\begin{array}{l}\text { S. } 8+\text { S. } 12 \text { na- } \\
\text { turel et viru- } \\
\text { lent }\end{array}$} & $\begin{array}{lr}\text { Echecs } & 193 \\
\text { Formes } & \\
\text { latentes } & 15\end{array}$ & $\begin{array}{r}198,22 \\
9,78\end{array}$ & 5,22 & $\begin{array}{l}27,25 \\
27,25\end{array}$ & $\begin{array}{l}0,14 \\
2,80\end{array}$ \\
\hline & $\begin{array}{l}\text { Total } \\
\text { souris } \\
\text { Total abcès } \\
\text { à formes } \\
\text { latentes } \quad 16\end{array}$ & & & & $x^{2}=7,62$ \\
\hline
\end{tabular}

Le $\varkappa^{2}$ est ici de 7,62. La différence est encore significative, même avec un seuil de confiance de $0,99\left(\gamma^{2}=6,64\right)$.

La différence qui apparait en comparant le nombre total des abcès se retrouve donc en comparant le nombre total des abcès à formes latentes.

B. SOURIS PRÉPARÉES.

1. Comparaison du nombre total d'abcès obtenus, d'une part avec la souche S. 8 non traitée ou traitée par un broyat tissulaire, d'autre part avec la souche S. 8 traitée 
préalablement par un homogénéisat de flagellés, S. 12 seul ou associé à un broyat tissulaire.

\section{Tableau 9}

\begin{tabular}{|c|c|c|c|c|c|c|}
\hline SouchE & \multicolumn{2}{|c|}{$\begin{array}{l}\text { RÉSULTATS } \\
\text { EXPÉRIMENTAUX }\end{array}$} & $\begin{array}{l}\text { VALEUR } \\
\text { THÉORIQUE }\end{array}$ & ECARTS & $\begin{array}{l}\text { CARRÉ DES } \\
\text { ÉCARTS }\end{array}$ & $\begin{array}{l}\text { CARRE des / VALEUR } \\
\text { ECARTS }\end{array}$ \\
\hline $\begin{array}{l}\text { S. } 8 \text { seul et S. } 8 \\
\text { + foie ou pan- } \\
\text { créas }\end{array}$ & $\begin{array}{l}\text { Echecs } \\
\text { Abcès }\end{array}$ & $\begin{array}{r}131 \\
3\end{array}$ & $\begin{array}{r}115,24 \\
18,76\end{array}$ & $\begin{array}{l}15,76 \\
15,76\end{array}$ & $\begin{array}{l}248,38 \\
248,38\end{array}$ & $\begin{array}{r}2,15 \\
13,24\end{array}$ \\
\hline \multirow[t]{2}{*}{ S. $8+$ S. 12} & $\begin{array}{l}\text { Echecs } \\
\text { Abcès }\end{array}$ & $\begin{array}{r}85 \\
3.2\end{array}$ & $\begin{array}{r}100,62 \\
16,38\end{array}$ & $\begin{array}{l}15,62 \\
15,62\end{array}$ & $\begin{array}{l}243,98 \\
243,98\end{array}$ & $\begin{array}{r}2,42 \\
14,89\end{array}$ \\
\hline & $\begin{array}{l}\text { Total } \\
\text { Souris } \\
\text { Total } \\
\text { abcès }\end{array}$ & $\begin{array}{r}251 \\
35\end{array}$ & & & & $x^{2}=32,70$ \\
\hline
\end{tabular}

$\mathrm{Ce} \%^{2}$ de 32,70 est tout à fait significatif, quel que soit le seuil de confiance choisi.

2. Comparaison du nombre d'abcès latents obtenus dans les deux mêmes lots que ci-dessus.

Tableau 10

\begin{tabular}{|c|c|c|c|c|c|c|}
\hline SOUCHE & \multicolumn{2}{|c|}{$\begin{array}{c}\text { RÉSULTATS } \\
\text { EXPÉRIMENTAUX }\end{array}$} & $\begin{array}{l}\text { VALEUR } \\
\text { THÉORIQUE }\end{array}$ & ECARTS & $\begin{array}{l}\text { CARRÉ DES } \\
\text { ÉCARTS }\end{array}$ & $\begin{array}{l}\text { CARRE des / VALEUR } \\
\text { ECARTS }\end{array}$ \\
\hline $\begin{array}{l}\text { S. } 8 \text { seul et S. } 8 \\
+ \text { foie ou pan- } \\
\text { créas }\end{array}$ & $\begin{array}{l}\text { Echecs } \\
\text { Formes } \\
\text { latentes }\end{array}$ & $\begin{array}{r}133 \\
1\end{array}$ & $\begin{array}{r}131,32 \\
2,68\end{array}$ & $\begin{array}{l}1,68 \\
1,68\end{array}$ & $\begin{array}{l}2,82 \\
2,82\end{array}$ & $\begin{array}{l}0,02 \\
1,05\end{array}$ \\
\hline \multirow[t]{2}{*}{ S. $8+$ S. 12} & $\begin{array}{l}\text { Echecs } \\
\text { Formes } \\
\text { latentes }\end{array}$ & $\begin{array}{r}113 \\
4\end{array}$ & $\begin{array}{r}114,66 \\
2,34\end{array}$ & $\begin{array}{l}1,66 \\
1,65\end{array}$ & $\begin{array}{l}2,75 \\
2,75\end{array}$ & $\begin{array}{l}0,02 \\
1,18\end{array}$ \\
\hline & $\begin{array}{l}\text { Total } \\
\text { Souris } \\
\text { Total à } \\
\text { formes } \\
\text { latentes }\end{array}$ & $\begin{array}{r}251 \\
5\end{array}$ & & & & $x^{2}=2,27$ \\
\hline
\end{tabular}

$\mathrm{Ce} \varkappa^{2}$ de 2,27 n'est pas significatif et nous ne retrouvons pas ici des résultats comparables à ceux offerts par les tableaux 7 et 8 . Il apparaît paradoxal d'envisager que le traitement préalable des souris puisse être mis en cause car il a été suffisamment montré, par ailleurs, qu'il améliorait le pourcentage de réussite. En revanche, il nous 
faut rappeler que cette dernière série d'expériences a été entreprise plus tardivement alors que les souches traitées en étaient à leur $18^{\circ}$ repiquage.

Ainsi sommes-nous amenés à constater le fait que la virulence conférée aux flagellés se trouve plus ou moins transitoire. Elle a disparu aux environs du $12^{\circ}$ repiquage. En comparant les résultats du tableau 9 et du tableau 10, on peut en conclure que la souche S. 8 , cultivée en présence d'homogénéisat de la souche $\mathrm{S} .12$, a manifestement acquis un pouvoir agressif vis-à-vis de la Souris comme le prouve le nombre total d'abcès observés: en revanche, ce pouvoir reste insuffisant pour se traduire par un nombre significatif d'abcès à formes latentes.

3. Comparaison du nombre total d'abcès obtenus, d'une part avec la souche S. 8 non traitée ou traitée par un broyat tissulaire, d'autre part avec les souches S. 8 provenant du développement in vitro d'abcès « à formes latentes ».

Tableau 11

\begin{tabular}{|c|c|c|c|c|c|c|}
\hline SOUCHE & \multicolumn{2}{|c|}{$\begin{array}{c}\text { RÉSULTATS } \\
\text { EXPÉRIMENTAUX }\end{array}$} & $\begin{array}{l}\text { VALEUR } \\
\text { THÉORIQUE }\end{array}$ & ECARTS & $\begin{array}{l}\text { CARRÉ DES } \\
\text { ÉCARTS }\end{array}$ & $\begin{array}{l}\text { CARRE des / VALEUR } \\
\text { ECA IS / thEORIQUE }\end{array}$ \\
\hline $\begin{array}{l}\text { S. } 8 \text { seul et S. } 8 \\
+ \text { foie ou pan- } \\
\text { créas }\end{array}$ & $\begin{array}{l}\text { Echecs } \\
\text { Abcès }\end{array}$ & $\begin{array}{r}131 \\
3\end{array}$ & $\begin{array}{l}127,3 \\
7,24\end{array}$ & $\begin{array}{l}3,7 \\
4,24\end{array}$ & $\begin{array}{l}13,7 \\
17,58\end{array}$ & $\begin{array}{l}0,11 \\
2,48\end{array}$ \\
\hline $\begin{array}{l}\text { S. } 8 \text { provenant } \\
\text { d'abcès à for- } \\
\text { mes latentes }\end{array}$ & $\begin{array}{l}\text { Echecs } \\
\text { Abcès }\end{array}$ & $\begin{array}{l}97 \\
10\end{array}$ & $\begin{array}{r}101,65 \\
5,78\end{array}$ & $\begin{array}{l}4,65 \\
4,22\end{array}$ & $\begin{array}{l}21,62 \\
17,81\end{array}$ & $\begin{array}{l}0,21 \\
3,08\end{array}$ \\
\hline & $\begin{array}{l}\text { Total } \\
\text { Souris } \\
\text { Total } \\
\text { abcès }\end{array}$ & $\begin{array}{r}241 \\
13\end{array}$ & & & & $\chi^{2}=5,88$ \\
\hline
\end{tabular}

Dans ce cas, la valeur du $\chi^{2}=5,88$ se trouve entre celle obtenue avec un seuil de 0,95 et un seuil de 0,99 . En considérant le nombre d'abcès, il apparaît que le passage chez l'animal n'entraîne pas, chez ces flagellés, l'acquisition d'un pouvoir virulent capable d'être ultérieurement transmis de génération en génération, à la différence des souches S. 12, S. 13, S. 14, comme nous l'avons déjà rapporté (2). Nous pouvons en conclure que le passage sur l'animal de Trichomonas vaginalis est une condition nécessaire mais non suffisante pour créer, chez une souche, une virulence transmissible héréditairement pendant une période plus ou moins longue.

Cette remarque se trouve confirmée par l'examen du résultat du tableau 12, il n'est alors nullement besoin de calculer le $\chi^{2}$ pour constater qu'il n'existe aucune différence entre les flagellés non modifiés (S. 8 seul et S. 8 + foie ou pancréas) et les flagellés prélevés à partir des abcès à formes latentes. 
Tableau 12

\begin{tabular}{|c|c|}
\hline SOUCHE & $\begin{array}{l}\text { RÉSULTATS } \\
\text { EXPÉRIMENTAUX }\end{array}$ \\
\hline S. 8 seul et S. $8+$ foie ou pancréas & $\begin{array}{lr}\text { Echecs } \ldots \ldots \ldots \ldots \ldots \ldots \ldots \ldots \ldots & 133 \\
\text { Formes latentes } \ldots \ldots \ldots \ldots \ldots \ldots & 1\end{array}$ \\
\hline S. 8 provenant d'abcès à formes latentes & $\begin{array}{lrr}\text { Echecs } & \ldots \ldots \ldots \ldots \ldots \ldots \ldots \ldots \ldots & 106 \\
\text { Formes latentes } \ldots \ldots \ldots \ldots \ldots \ldots & 1\end{array}$ \\
\hline & $\begin{array}{lr}\text { Total Souris . ................. } & 241 \\
\text { Total abcès à formes latentes ..... }\end{array}$ \\
\hline
\end{tabular}

VI. Conclusions.

A) En ce qui concerne l'endoparasitisme expérimental chez la Souris, la souche S. 8 présente un comportement biologique particulier puisqu'elle se montre totalement réfractaire.

B) Le fait que cette souche ait pu donner, en 1961, des résultats favorables nous permet de conclure que le vieillissement provoqué par une culture prolongée in vitro peut être rendu responsable de la disparition d'un caractère que l'on retrouve chez les souches plus jeunes.

C) Ce caractère une fois perdu semble difficile à récupérer.

1. L'addition d'un broyat de tissu, foie ou pancréas, pour lequel le parasite sem. ble montrer une affinité évidente n'entraîne aucune modification de la virulence de la souche S. 8, contrairement aux espoirs suscités par certains résultats encourageants obtenus à partir d'autres souches beaucoup plus jeunes.

2. Dans notre travail, seule, l'addition d'un homogénéisat de flagellés de même espèce et de souche différente provoque une certaine exaltation provisoire du caractère agressif. Le mécanisme intime de l'acquisition d'un tel caractère demeure inexpliqué. Le fait est pourtant remarquable et des travaux en cours visent à approfondir cette voie.

a) Cette exaltation se traduit par la possibilité d'implantation chez l'animal sans que puissent être observées des lésions aussi sévères que celles habituellement décrites.

b) A notre avis, au cours de leur passage chez l'animal, les flagellés de la souche S. 8 peuvent présenter, au sein des abcès à formes latentes, des formes de survie ou de résistance révélées par la culture ultérieure de ces abcès.

D) Le séjour chez l'animal n'est pas une condition suffisante pour obtenir l'exaltation durable du caractère virulent comme le prouvent le résultat des réinoculations effectuées à partir des abcès à formes latentes. 


\section{Bibliographie}

1. Georges (P.), 1964. - Etude expérimentale sur le comportement biologique de différentes espèces du genre Trichomonas. Thèse Doctorat Pharmacie (Etat) Paris.

2. Gobert (J.-G.), Georges (P.), Gobert (N.) et Savel (J.), 1969. - Etude de l'endoparasitisme expérimental de Trichomonas vaginalis chez la Souris. I. Conditions d'installation et obtention de lésions viscérales avec diverses souches du flagellé. Ann. Parasitol. hum. comp. (sous presse).

3. Gobert (J.-G.), Georges (P.), Savel (J.), Genet (P.) et Piette (M.), 1969. - Etude de l'endoparasitisme expérimental de Trichomonas vaginalis chez la Souris. II. Etude cytologique et histologique. Ann. Parasitol. hum. comp. (sous presse).

4. INOKI (S.) et Hamada (Y.), 1953. - Experimental transmission of Trichomonas vaginalis (pure culture) into mice. J. infect. dis. 92, 1-3.

5. Magara (M.), Amino (E.) et Youkouti (E.), 1953. - One method for the pure culture of Trichomonas vaginalis. Amer. J. Trop. Med. Hyg., 2, 267-269.

6. Résultats inédits. 